\title{
Forthcoming Challenges due to COVID-19 Pandemic Caused by SARS-CoV-2 Novel Coronavirus
}

\author{
Mohit Bhardwaj, Prem Kumar and Adil Jokhi, \\ Department of Pulmonary Medicine Shree Guru Gobind Singh \\ Tricentary University, Gurugram, Haryana, India \\ Corresponding author email: bhardwajmohit007@rediff.com
}

\section{ABSTRACT}

Pandemic of COVID-19 triggered by SARS-COV-2 now spread all over the world. Government and medical authorities are trying to manage this respiratory disease efficiently. Due to lack of essential supply, many sickbays are distress from paucity of sanitizing liquid, protecting masks as well asfree beds. For defeating this disease, it is necessary to come all countries togetherand coordinate with each other. Various challenges may come in the future, the major challenge may be the development of effective COVID-19 vaccine in order to deliversafeguardas well asinterfere the transmission of SARS-CoV-2 and the distribution of the vaccine. Internationally, it must be ensured thatthe vaccine should reach to every person. The manufacturing of the vaccine on mass scale is also a challenge. According to the population of the world, due to limited number of vaccine manufacturing companies, it will take so much time to manufacture the vaccine for reaching out to every person. The present paper mainly discussed about the upcoming challenges due to the COVID-19 pandemic that will face by the world in the future.

KEY WORDS: COVID-19, HEALTHCARE, PANDEMIC, SARS-COV-2, VACCINE.

\section{INTRODUCTION}

The SARS-COV-2 virustriggers the pandemic of COVID-19 has affected more than 7.5 million people worldwide and 1.15 lakh deaths. This respiratory disease spread over almost 200 countries, most of the cases reported from Brazil, United states of America (USA), India and Russia(J. J. Ramsden et. al. (2020)). The numbers of cases are still increasing day by day. The countries like South Korea, New Zealand and China has already flattened the curve of COVID-19 cases. On the other hand, some countries are still struggling to control the novel corona virus cases. Every country's government authority and medical authorizes put their extrastruggles to control situation(T. P. Velavan et. al. (2020)). There are various challenges are facing by many countries such as lack

Biosc Biotech Res Comm P-ISSN: 0974-6455 E-ISSN: 2321-4007
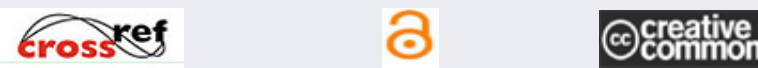

Identifiers and Pagination

Article Information

Year: 2021 Vol: 14 No (6) Special Issue

Pages: $330-333$

Received: $08^{\text {th }}$ May 2021

This is an open access article under Creative

Commons License Attribn 4.0 Intl (CC-BY).

DOI: $h t t p: / / d x . d o i . o r g / 10.21786 / b b r c / 14.7 .70$ of free beds, personal protection equipment (PPE) kits and protective masks. These challenges can cause of community spread of viral infection.

In order to control this respiratory disease triggered by SARS-COV-2 virus, the efforts must be taken at global level. Every country needs to coordinate with each other and provide the essential supply to help other countries. Apart from the efforts taken by government authorities, every person must be follow the preventive measures such as social distancing, wash hands frequently and weak facemask to avoid the transmission of novel corona virus(WHO et. al. (2020)). There will be some challenges in front of the world that has to be tackle in the future; the major challenge will be development of effective COVID-19 vaccine. Many researchers from all over the world are trying to develop long term effective COVID-19 vaccine;some already developed vaccines are in different phases of testing. Some other challenges are psychological and physiological changes due to COVID-19 pandemic. Various cases of depression have been reported that caused due to COVID-19 pandemic(Y. C. Wu et. al. (2020)). 
In the pandemic situation, it is necessary to follow the preventive measures suggested by government and medical authorities until effective drug or vaccine is not available for the viral infection. Because of social disconnect in viral infection pandemic situation, neurological and physiological changes could be possible. This review article provides an overview of COVID-19 pandemic, mainly discussed about the transmission and prevention of novel coronavirus and future challenges due to the pandemic of COVID-19. This evaluation article delivers good references to the researchers about forthcoming challenges caused by novel coronavirus and how these challenges can be handle efficiently.

History Of Coronavirus Outbreaks: From the last 100 decades, coronaviruses repeatedly spread worldwide(D. Forni et. al. (2017)). The first coronavirus, called bronchitis virus (IBV), was identified and discovered due to the illness from barnyard fowl in thirtiesfollowed by hepatitis viruses from rats in forties(F. R. Beaudette et. al. (1937)). In 1946 at United States of America (USA), a virus found in pigs named transmissible gastroenteritis virus (TGEV). The first human coronavirus was identified in 1960 from the respiratory tract infection. $229 \mathrm{E}$ and B814 were the first two isolated viruses(D. A. Tyrrell et. al. (1966)). The different coronaviruses are identified continuously in humansand the numbers are significantly increasing. These viruses are spread from different animal species including bats, dogs, turkeys and calves.

In 2002-2003, SARS-CoV coronaviruswas widely spread in 30 countries and maximuminfected patients were found in China as well as Hong Kong. Total 8096 cases have been reported and 774 out of them were died, the corresponding fatality rate was 9.6\%(World Health Organization et. al. (2004)). SARS-CoV had very closed characteristics to another virus comes from palm civets, based on genome sequence. After that, bats acts as a natural host and civets were played a role ofmiddle host for the SARS-CoV coronavirus(J. Cui et. al. (2019)). In a recent research, a team of researchers identified 11 SARS-like CoVs clviruses from horseshoe bats in Yunnan province, China. Based on the genome sequence, high genetic diversity found out in these viruses. All viruses had different $\mathrm{S}$ protein sequences but still showing close characteristics with SARS-CoV. Several cases of viral infection caused by SARS-CoV coronavirus have been identifiedand the characteristics of the SARS-CoV coronavirus were similar to the other viruses that come from the Himalayan palm civets.

After one decade, a MERS-CoV virus identified that transmitted to the human from the camel. Most of the Middle Eastern countries were affected due to MERS$\mathrm{CoV}$ virus. The epidemic caused by MERS-CoV virus has confirmed 2519 cases and 866 deaths till January of 2020. Phylogentically, MERS-CoV shows the very close characteristics to the HKU4 and HKU5 coronavirus that comes from the bat. In the December of 2019, novel SARS-CoV-2 coronavirus appeared in Wuhan in China that cause criticalbreathingsickness as well as mortality. Approximately 200 countries are affected by this virus till now. Early research stated that it might be originates from the bats. Different coronaviruses evolves from last two decades and all coronaviruses showed similar characteristics based on genome sequence.

Symptoms Of The Covid-19: The sign and indications of the COVID-19 infection might appear in two to 15 days after contact. The time span between contacts to coronavirus as well as when indications start appearing generally known asthe incubation span. In some cases, symptoms may take more time to appear. That is why it is necessary to isolate himself from other person when people come in contact with infected person or have exposure to the place where viral infection cases have been found.There are some common sign and symptoms are:

\section{- Tiredness \\ - Cough \\ - Fever}

COVID-19 can be impaired by a lack of taste or smell in the beginning. Additional signs can include:

- Runny nose

- Chills

- Sore throat

- Headache

- Chest pain

- Muscle aches

- Shortness of breath or difficulty in breathing

There are various sign and symptoms are associated with the COVID-19 infection triggered by SARS-CoV-2. When early symptoms of COVID-19 infection found in any person, the preventive measure (avoid social contact, use facemask while coughing and sneezing etc.) must be follow by the person until COVID-19 testing has not been done. In case of severe symptoms like cough, fever and difficulty in breathing, contact government and medical authority for the medical attention.

\section{Transmission And Prevention Of Novel Coronavirus Sars-Cov-2}

1. Mode of Spreading: COVID-19 is a respiratory infectious disease. People can get infected by virus if any person comes in close contactwith the infected person who has indications of infection such as cough as well as sneezing. In general, the transmission of coronavirus is happening due to the droplets, the sizes of these droplets are typically $>5-10 \mu \mathrm{m}$ in diameter. These droplets are called respiratory droplets. When the diameter of the droplets $<5$ $\mu \mathrm{m}$, referred as nuclei droplets. Due to the bigger size of the respiratory droplet, the lifespan of these droplets are very small and it cannot travel more than 1 meter in the air.

The other mode of transmission is through airborne. Airborne transmission is different from droplet transmission, and airborne transmission caused by particles or units less than $5 \mu \mathrm{m}$ in dimension of diameter. These particles persist in the surroundings for longer time as well as capable of travelling over long distance ( $>1$ meter). The size of the airborne are very small, it appears in the air while coughing and sneezing along with the respiratory droplets. Because of smaller size, it can travel more than one meter in the air. 
2. Prevention: Currently, the vaccines are unavailable to guard from COVID-19. In order to protect from COVID19 , every person is to evade being uncovered to virus that triggers COVID-19. In order to prevent spreading of coronavirus, every person needs to take some preventive measures as shown in Figure 1.

Figure 1: Schematic illustration of the Preventive measures in order to restrict the Transmission of the Coronavirus

Preventive Measures for

Transmission of coronavirus

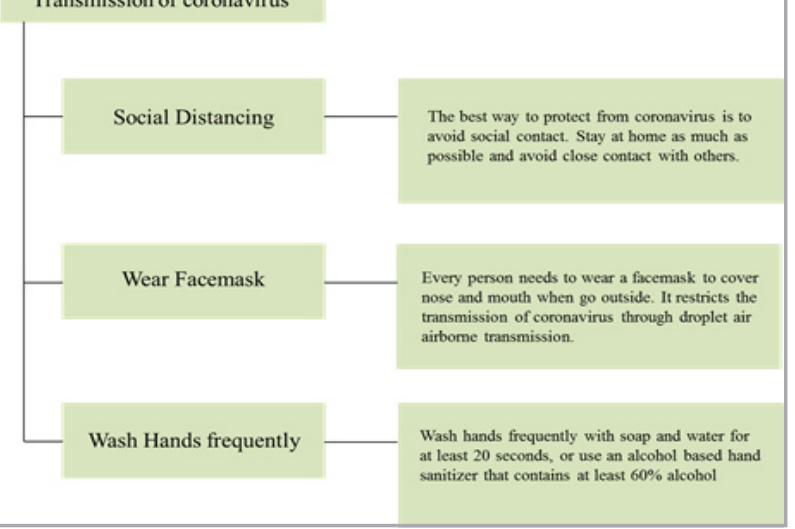

3. Management and Vaccination: Many researchers from all over the world are working for the development of effective COVID-19 vaccine. Most of the healthcare professionals are gives supportive therapy to the patients such as hydration maintenance, analgesic and antipyretic administration and mechanical ventilation. In some cases antibiotic gives to the patients in the case of bacterial infections. In order to suppress the transmission of the COVID-19 infection triggered by SARS-CoV-2, only the effective vaccine and drug can provide a reliable solution for this pandemic situation.

\section{Forthcoming Challenges Due To Covid-19 Pandemic Caused By Sars-Cov-2 Novel Coronavirus}

1. Development of Vaccine: For treating the COVID19, antiviral agents and vaccine will be remains the first priority from the edpidemiological and microbiological point of view. Currently, there is no effective and specific treatment is available against the COVID-19 viral infection. In recent research, it is reported that remdesivir shows the effective results for treating the coronavirus and could be the most promising agent that can help to minimize the criticality of the patients suffering from COVID-19. These results are comes from the initial trials, in order to validate, further inspectionsas well as clinical trials are need to be perform. In last few months, there are various drugs have been inspected for the cure of the COVID-19 infection but in order to ensure health safety, further clinical trials needs to be done.

There are various laboratories are working in the field of development of vaccine for COVID-19. But till now, now therapeutic or prophylactic vaccine thatauthenticated yet. Some laboratories are doing research on at least eightpromising vaccines(A. Kumar et. al. (2020)). Few research groups are trying to develop measles vaccine that can help to provide antigens that may induce the protection against the COVID-19. In order to control the pandemic due to COVID-19, progress of effective vaccine or antiviral medication will take many months. Because of the difficulty to understand about genomic recombination, researchers are hesitant to speak in the development of antiviral medicines or any preventive vaccine about guidelines of these approaches. Therefore, the researchers from new generation need to devote specifically to the molecular aspects of the viral pathogenesis and try to gain knowledge about the genome sequencing of the isolates.

2. Fast COVID-19 Diagnosis tests: Another challenge in front of the healthcare professionals is rapid diagnosis of COVID-19. Currently, the diagnosis of the infectious disease usually takes longer time. In developed countries, RT-PCR and serological test are used for speedy diagnosis but because of limited access of these diagnosis assays in the low socioeconomic region, developing countries are facing some problems. Internationally, it must be ensure that fast diagnosis assays must be provided to developing countries that has large number of COVID-19 cases. The availability of rapid diagnosis kits can help to restrict the transmission of COVID-19.

3. Lack of Essential necessities: When the number patients increase rapidly, the supply of the essential needs is very important. The admitted patients' to the hospitals needs thebeds, facemasks and sanitizing liquid. Apart from these things, more number of personal protection equipment (PPE) kits and healthcare professionals required. For the developing countries, it is very difficult to supply all essential necessities things in time. This can cause the increase number of cases. For health services around the globe, this is a big problem. Many countries already have adequate ICU beds as well machines of ECMO and safe and clear shelter for the admission of new patients. This issue demanded urgent actions to prevent a spike in COVID-19 incidents.

\section{CONCLUSION}

The COVID-19 cases are is still showing the exponential growth in some countries and few countries controlled the transmission of coronavirus and flattened curve of the cases of this respiratory disease. No effective vaccine has been reported yet for the COVID-19 treatment. That is why there are some preventive measures that need to be followedby every person in order to avoid the transmission of coronavirus. This viral disease spread almost all over the world and it will take several months to overcome such problem until the proper vaccine or antiviral drug will develop. Due to this life-threaten pandemic situation, there are various challenges are in front of the world such as development of effective preventive vaccine or antiviral drug and rapid test kit for COVID-19. Developing countries are still facing problem of limited access of fast diagnosis kits due to low socioeconomic level. In the future, in order to handle this epidemic situation, all countries must come together and coordinate with each other to help developing countries. 


\section{REFERENCES}

Beaudette, F.R., 1937. Cultivation of the virus of infectious bronchitis. J. Am. Vet. Med. Assoc., 90, pp.51-60.

Case, D.A., Darden, T.A., Cheatham III, T.E., Simmerling, C.L., Wang, J., Duke, R.E., Luo, R., Walker, R.C., Zhang, W., Merz, K.M. and Roberts, B., 2010. AMBER 11, University of California, San Francisco. Google Scholar There is no corresponding record for this reference.

Forni, D., Cagliani, R., Clerici, M. and Sironi, M., 2017. Molecular evolution of human coronavirus genomes. Trends in microbiology, 25(1), pp.35-48.

Goniewicz, K., Khorram-Manesh, A., Hertelendy, A.J., Goniewicz, M., Naylor, K. and Burkle, F.M., 2020. Current response and management decisions of the European Union to the COVID-19 outbreak: a review. Sustainability, 12(9), p.3838.

Kumar, A., Saxena, A.K., Lee, G.G.C., Kashyap, A. and Jyothsna, G., 2020. Identification of potential Vaccine
Candidates for COVID19. In Novel Coronavirus 2019 (pp. 67-74). Springer, Singapore.

Tyrrell, D.A.J. and Bynoe, M.L., 1966. Cultivation of viruses from a high proportion of patients with colds. Lancet, pp.76-7.

Velavan, T.P. and Meyer, C.G., 2020. La epidemia de COVID-19. Trop Med Int Health, 25(3).

World Health Organization, 2003. Summary of probable SARS cases with onset of illness from 1 November 2002 to 31 July 2003. http://www. who. int/csr/sars/country/ table2004_04_21/en/index. html.

Wu, C., Yi, Chen. Sung, Ching. Chan. Jiun, Yu.(2020). The outbreak of Covid-19: An overview. Journal of the Chinese Medical Association, 83(3), pp.217-220.

Zhou, P., Yang, X., Wang, X., Hu, B., Zhang, L., Zhang, W., Si, H., Zhu, Y., Li, B., Huang, C. and Chen, H., 295 Luo Y. Guo H, Jiang R, Liu M, Chen Y, Shen X, Wang X, Zheng X, Zhao K, Chen Q, Deng F, Liu L, 296, pp.270273. 